\title{
Preliminary evidence of a noncausal association between the X-chromosome inactivation pattern and thyroid autoimmunity: a twin study
}

\author{
Thomas Heiberg Brix ${ }^{\star, 1}$, Pia Skov Hansen ${ }^{1,3}$, Kirsten Ohm kyvik ${ }^{2,3}$ and Laszlo Hegedüs ${ }^{1}$
}

An increased frequency of skewed X-chromosome inactivation $(\mathrm{XCI})$ is found in clinically overt autoimmune thyroid disease (AITD) compared with controls. Whether skewed $\mathrm{XCI}$ is involved in the pathogenesis of autoantibodies to thyroid peroxidase (TPOAb) in euthyroid subjects is unknown. To examine the impact of $\mathrm{XCl}$ on the serum concentration of TPOAb, we studied whether within-cohort and within-twin-pair differences in $\mathrm{XCl}$ are associated with differences in serum concentrations of TPOAb. A total of 318 euthyroid female twin individuals distributed in 159 pairs were investigated. XCI was determined by PCR analysis of a polymorphic CAG repeat in the first exon of the androgen receptor gene. TPOAb concentrations were measured using a solid-phase time-resolved fluoroimmunometric assay. Overall (within cohort), there was a significant association between $\mathrm{XCI}$ and serum concentrations of TPOAb; regression coefficient $(\beta)=1.45$ (95\% confidence interval, $0.52-2.38), P=0.003$. The association remained significant in the within-pair analysis; $\beta=1.74(0.79-2.69), P<0.001$. The relationship was nonsignificant within the 82 monozygotic pairs $(\beta=0.57(-0.78-1.92), P=0.405)$, whereas the association was significant in the 77 dizygotic pairs $(\beta=2.17(0.81-3.53), P=0.002)$. This preliminary finding of a significant association between TPOAb concentrations and $\mathrm{XCl}$ within cohort and within dizygotic but not within monozygotic twin pairs may indicate that $\mathrm{XCl}$ per se does not have a major role in the pathogenesis of TPOAb. More likely, $\mathrm{XCI}$ and TPOAb are influenced by shared genetic determinants.

European Journal of Human Genetics (2010) 18, 254-257; doi:10.1038/ejhg.2009.156; published online 30 September 2009

Keywords: X-chromosome inactivation; thyroid peroxidase antibodies; thyroid autoantibodies; thyroid autoimmunity; twins; epigenetics

\section{INTRODUCTION}

Clinically overt autoimmune thyroid diseases (AITD) affect around $2 \%$ of the female population, whereas prevalence of subclinical disease, reflected by the presence of autoantibodies against thyroid peroxidase (TPOAb) and thyroglobulin in euthyroid individuals, is up to 10 -fold higher. ${ }^{1,2}$ Although common, the aetiology of these diseases is still incompletely understood. However, it is generally assumed that the development of overt as well as subclinical AITD is the net result of environmental triggers ${ }^{3-5}$ operating in genetically predisposed individuals. ${ }^{6-9}$ Recently, epigenetic factors such as genetic anticipation $^{10}$ and the phenomenon of X-chromosome inactivation $(\mathrm{XCI})^{11-13}$ have been suggested to have a role in the aetiology of AITD. $\mathrm{We}^{11}$ and subsequently others ${ }^{12,13}$ have reported an association between a skewed XCI pattern and the presence of clinically overt AITD in females. Thus, there is little doubt that an association exists between skewed XCI and overt AITD. The key question, however, is whether it is the skewed XCI per se or other factors such as genes, socioeconomic or environmental conditions that cause the observed association.

Because monozygotic (MZ) twin pairs share $100 \%$ of their genes and dizygotic (DZ) pairs share 50\% (on average), studies of twins offer a unique opportunity to distinguish between genetic and nongenetic influences. ${ }^{14,15}$ It follows that differences within DZ pairs are because of a combination of genetic and nongenetic factors, whereas differences within MZ pairs are caused by nongenetic factors. ${ }^{14,15}$ Thus, if the association between skewed XCI and AITD is because of genetic confounding (the genotype responsible for AITD is also involved in the XCI pattern), the association would be observed within DZ pairs, but not within MZ pairs. In this study, we examined the relationship between the serum concentrations of TPOAb and the degree of skewed X-chromosome inactivation in 159 euthyroid female twin pairs.

\section{SUBJECTS}

The twins were recruited from the The Danish Twin Registry. ${ }^{16}$ A detailed description of the ascertainment procedure has been published elsewhere. ${ }^{17}$ In brief, in 1997, a representative sample of self-reported healthy twin pairs born between 1931 and 1982 was recruited from the Danish Twin Register on the basis of nationwide questionnaire surveys regarding health and health-related behaviour conducted in 1994 and 1996. In all, 1512 individuals (756 twin pairs) were examined from 1997 to 2000. Blood samples were available from 736 twin pairs. Twin pairs with self-reported thyroid disease (32 subjects in 28 twin pairs) or overt biochemical thyroid disease (19 subjects in 18 pairs) were excluded. Moreover, all males (688 subjects) and females from opposite-sex pairs (120 subjects) were also excluded, leaving 572 females (286 pairs). Of these, 318 subjects (159 twin pairs, distributed in $82 \mathrm{MZ}$ and $77 \mathrm{DZ}$ pairs) were

${ }^{1}$ Department of Endocrinology and Metabolism, Odense University Hospital, Odense, Denmark; ${ }^{2}$ Institute of Regional Health Services Research, University of Southern Denmark, Odense, Denmark; ${ }^{3}$ The Danish Twin Registry, Epidemiology, Institute of Public Health, University of Southern Denmark, Odense, Denmark

${ }^{*}$ Correspondence: Dr TH Brix, Department of Endocrinology, Odense University Hospital, Sdr. Boulevard 29, 5000 Odense C, Denmark.

Tel: +45 654117 10; Fax: + 45659069 38; E-mail: thomas.brix@ouh.regionsyddanmark.dk

Received 9 January 2009; revised 30 June 2009; accepted 10 August 2009; published online 30 September 2009 
informative regarding both TPOAb and XCI pattern and hence suitable for data analysis. Informed consent was obtained from all participants, and the study was approved by all the Regional ScientificEthical Committees in Denmark.

\section{METHODS}

Assays

X-chromosome-inactivation analysis. DNA was extracted from peripheral blood cells. The X-chromosome phenotype was determined by polymerase chain reaction (PCR) analysis of a polymorphic repeat in the first exon of the androgen receptor gene. ${ }^{18}$ After digestion of the DNA with the methylationsensitive enzyme Hpall, a PCR product is obtained from the inactive $\mathrm{X}$ chromosome only. The PCR products were separated on an ABI 3100 automated sequencer, and analysed by GeneScan software (Applied Biosystems, Foster City, California, USA). Each sample was analysed in duplicate and blinded as to the result in the corresponding co-twin and the TPOAb phenotype. XCI was calculated as the percentage of the predominantly inactive allele to the sum of both alleles and varies between 50 and 100, where 50 reflects random XCI and 100 reflects a completely skewed XCI.

Serum TSH and TPOAb. Serum concentrations of TSH and TPOAb were measured using solid-phase time-resolved fluoroimmunometric assays (AutoDELFIA, Perkin-Elmer/Wallac, Turku, Finland).

Zygosity determination. Zygosity was established by DNA fingerprinting using a PE Applied Biosystems AmpFISTER Profiles Plus Kit (Foster City, California, USA).

Statistical methods. Group frequencies were compared with the Pearson $\chi^{2}$-test, whereas group medians were compared with a two-sample Wilcoxon rank-sum (Mann-Whitney) test using Brunner's adjustment for clustering within twin pairs. ${ }^{19}$ Because of deviation from normality, logarithmic transformation of TSH, TPOAb and XCI was carried out. The relationship between the logged values of XCI and TPOAb (within-cohort analysis), as well as the relationship between the within-twin-pair differences in XCI and within-pair differences in TPOAb (within-twin-pair analysis), was assessed by linear regression.

In the within-cohort analysis, the paired nature of twin data was taken into account by using only one, randomly chosen twin individual from each twin pair. Subsequently, data were analysed with the logged concentration of TPOAb as the response variable and $\ln \mathrm{XCI}, \operatorname{lnTSH}$, age, smoking and zygosity as explanatory variables. In the within-twin-pair analysis, the regression line was constrained to pass through the origin so that the results were independent of the labelling of the twin as the first or second.

Significant differences were defined as a P-value less than 0.05 using two-tailed tests. All analyses were carried out using version 7 of the STATA statistical package.

\section{RESULTS}

Descriptive characteristics and distribution of TPOAb and XCI measurements in the twin cohort, as a whole and stratified by zygosity, are given in Table 1. Statistically significant differences between MZ and DZ twins were observed for age (MZ vs DZ; 35 vs 38, $P=0.022$ ) and serum TSH (MZ vs DZ; 1.75 vs 1.44, $P=0.017$ ).

The results of the regression analyses are outlined in Figure 1 and Table 2. In the within-cohort analysis, a positive association between the serum concentration of TPOAb and XCI was found: regression coefficient $(\beta)=1.45$ (95\% confidence interval, $0.52-2.38$ ), $P=0.003$. As evident from Table 2, adjusting for age, TSH, smoking and zygosity did not significantly change the association. The association between $\mathrm{TPOAb}$ and XCI remained statistically significant in the overall within-pair analysis (both $\mathrm{MZ}+\mathrm{DZ}): \beta=1.74(0.79-2.69), P<0.001$. The relationship was nonsignificant within the $82 \mathrm{MZ}$ pairs $(\beta=0.57$ $(-0.78-1.92), P=0.405)$, whereas the association was significant in the $77 \mathrm{DZ}$ pairs $(\beta=2.17(0.81-3.53), P=0.002)$. Testing the equality of the within-pair regression coefficients across zygosity indicated that the associations were different between $\mathrm{MZ}$ and $\mathrm{DZ}$ twins $(P=0.07)$.

\section{Table 1 Basic characteristics}

\begin{tabular}{lccc}
\hline Variable & \multicolumn{3}{c}{ Study population } \\
& $M Z+D Z(n=318)$ & $M Z(n=164)$ & $D Z(n=154)$ \\
\hline Smoking (\%) & 52 & $48^{\mathrm{a}}$ & 56 \\
Age (year) & $36(19-51)$ & $35(19-51)^{\mathrm{b}}$ & $38(21-52)$ \\
Degree of skewing & $63(51-87)$ & $63(51-87)^{\mathrm{a}}$ & $63(51-88)$ \\
TPOAb (kIU/l) & $4.7(2.2-93.0)$ & $4.6(2.3-79.6)^{\mathrm{a}}$ & $4.9(2.8-156.3)$ \\
TSH (mU/l) & $1.56(0.65-3.34)$ & $1.75(0.73-3.55)^{\mathrm{c}}$ & $1.44(0.61-3.22)$
\end{tabular}

All values, except for smoking, are presented as medians with 5th and 95th percentiles in parentheses. MZ, monozygotic and DZ, dizygotic.

${ }^{\mathrm{M} Z Z}$ vs DZ, $P>0.05$

bMZ vs DZ, $P=0.022$

MZ vs DZ, $P=0.017$

As evident from Figure 1, the within-pair difference in lnTPOab and $\operatorname{lnXCI}$ among MZ twins is quite small. Theoretically, this small variability could mask a genuine association. To overcome this limitation, we restricted the analyses to $\mathrm{MZ}$ pairs with the highest degree of within-pair variation in $\operatorname{lnTPO}$ and $\operatorname{lnXCI}$. The regression coefficient was, however, still nonsignificant (MZ pairs with a within-pair difference in lnTPOAb of at least $0.5: n=17, \beta=4.58(-4.55-13.71)$, $P=0.303$. MZ pairs with a within-pair difference in $\operatorname{lnXCI}$ of at least $0.1: n=22, \beta=0.35(-0.23-0.94), P=0.224)$.

\section{DISCUSSION}

A link between a skewed XCI pattern and the development of clinically overt AITD in females has been suggested. ${ }^{1-13}$ In accordance with these studies, we found a statistically significant positive association between XCI and the serum concentrations of TPOAb in euthyroid subjects (within-cohort analysis). To evaluate whether this association was causal or could be explained by the presence of genetic or environmental confounders, we also analysed the association between $\mathrm{XCI}$ and TPOAb within MZ and DZ pairs. Despite similar distributions of XCI and TPOAb between MZ and DZ twins, stratification according to zygosity had a major impact on the results. The association was significant within the DZ pairs, whereas the relationship was nonsignificant within $\mathrm{MZ}$ pairs. Interaction analysis showed that the associations tended to be significantly different between $\mathrm{MZ}$ and $\mathrm{DZ}$ pairs $(P=0.07)$, indicating that genetic factors could have a major role in the association between the variance in XCI and that in the serum concentration of TPOAb. In other words, XCI per se does not seem to have a role in the aetiology of early AITD. More likely, XCI and AITD are influenced by common genetic determinants. The XCI process is under genetic control ${ }^{20}$ and it has been linked to loci on the $\mathrm{X}$ chromosome. ${ }^{21}$ The $\mathrm{X}$ chromosome may also be of interest in AITD because several genes crucial for the maintenance of immune function and tolerance are located on this chromosome. ${ }^{22}$ Linkage as well as association between genes on the $\mathrm{X}$ chromosome and AITD has been demonstrated. ${ }^{23-25}$ Thus, it is likely that the association between XCI and AITD is not causal but just a reflection of the inheritance of common X-linked susceptibility genes. In other words, our preliminary data indicate that the XCI pattern does not per se influence the serum concentration of TPOAb.

Our study has potential limitations. Approximately two-thirds of MZ twins are monochorionic (common placenta), whereas almost all DZ pairs are dichorionic. Therefore, it could be argued that besides genetic factors, intrauterine factors may also differ between $\mathrm{MZ}$ and DZ twins and this could theoretically be the cause of the observed difference between $\mathrm{MZ}$ and $\mathrm{DZ}$ pairs in the within-pair associations 

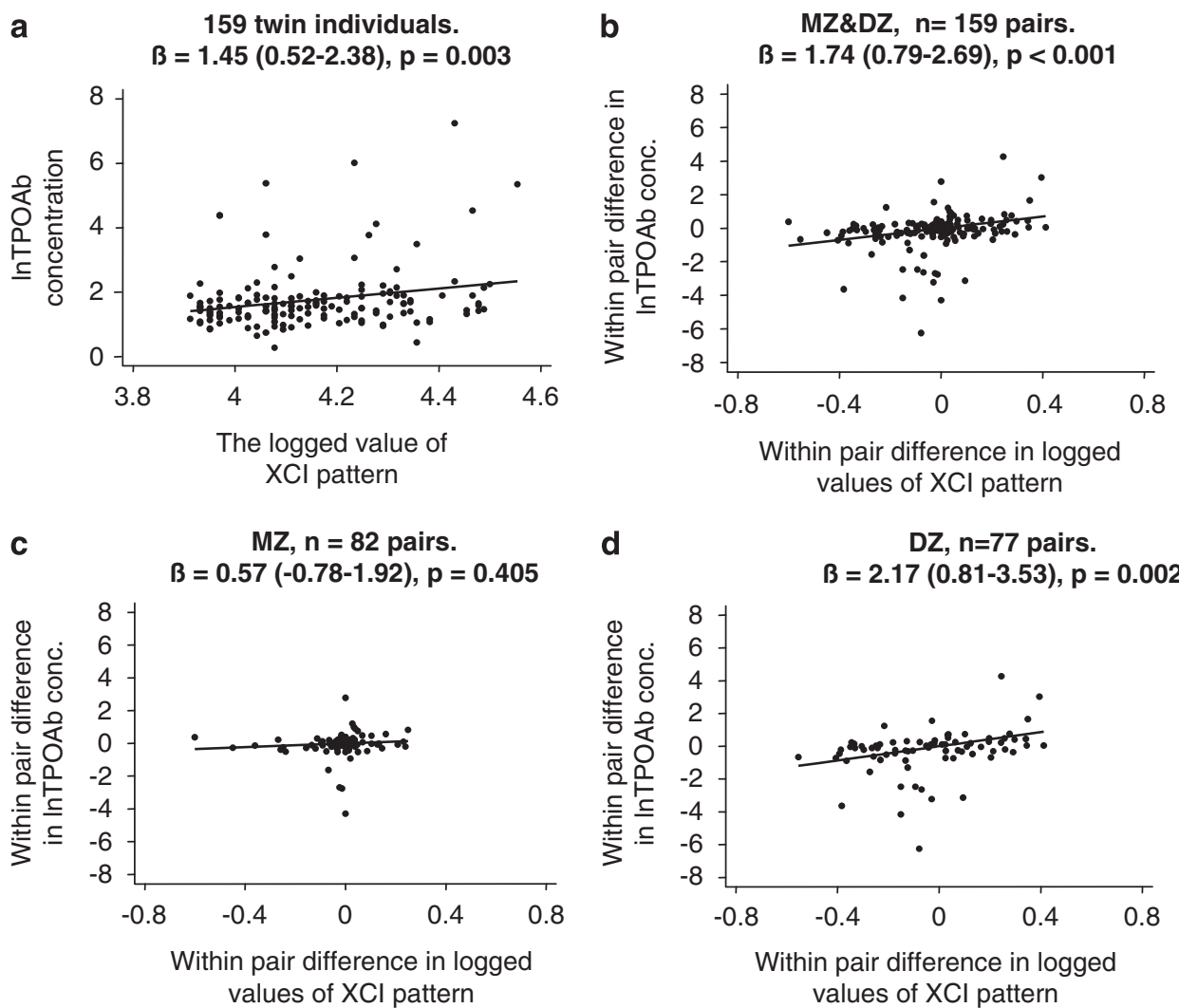

Figure 1 Scatterplots and unadjusted regression coefficients $(\beta)$ for the correlation between the concentration of TPOAb and the X-chromosome-inactivation (XCl) pattern for the within-cohort (a) and within-twin-pair (b, $\mathbf{c}$ and $\mathbf{d}$ ) comparisons. Ninety-five percent confidence intervals are given in parentheses.

Table 2 Regression coefficients between the $X$ chromosome inactivation pattern and serum concentrations of TPOAb

\begin{tabular}{|c|c|c|}
\hline \multirow[t]{2}{*}{ Analysis } & \multicolumn{2}{|c|}{ Regression coefficients (95\% confidence intervals) } \\
\hline & Unadjusted & Adjusted \\
\hline Within cohort & $1.45(0.52-2.38)$ & $1.40(0.45-2.35)^{\mathrm{a}}$ \\
\hline Within twin pair & $1.74(0.79-2.69)$ & $1.49(0.53-2.44)^{\mathrm{b}}$ \\
\hline Monozygotic pairs & $0.57(-0.78-1.92)$ & $0.43(-0.89-1.73)^{\mathrm{b}}$ \\
\hline Dizygotic pairs & $2.17(0.81-3.53)$ & $1.98(0.56-3.39)^{\mathrm{b}}$ \\
\hline
\end{tabular}

adjusted for age, TSH, smoking habits and zygosity.

bAdjusted for age, TSH and smoking habits.

between XCI and TPOAb. In our study, as in most twin studies, information on the anatomy of chorion and placenta was not available.

All participants were twins, but there is no reason to suspect that XCI pattern and the factors influencing TPOAb differ between twins and singletons. Available studies have clearly shown that Danish twins are representative of the general background population for an XCI pattern ${ }^{20}$ and for a range of thyroid phenotypes, ${ }^{6,7,26}$ including for the presence of TPOAb. ${ }^{9}$

Finally, because of the complete match for genetic factors, the within-pair difference in the concentration of TPO antibodies is quite narrow within MZ twins. Thus, our finding of a nonsignificant regression coefficient within $\mathrm{MZ}$ pairs could be because of a lack of statistical power. In fact, the $R^{2}$ in the unadjusted regression model within MZ pairs was only 0.009 . This means that over $1000 \mathrm{MZ}$ twin pairs would be needed to detect a significant difference at this level with a power of at least $90 \%$. In our view, a difference at this level is hardly likely to be of any clinical significance.

In conclusion, our demonstration of a significant association between TPOAb concentrations and XCI in the overall twin population and within $\mathrm{DZ}$ but not within $\mathrm{MZ}$ twin pairs, if confirmed in larger studies, suggests that XCI per se does not have a major role in the pathogenesis of TPOAb. Rather, the XCI pattern and TPOAb levels are influenced by shared genes.

\section{ACKNOWLEDGEMENTS}

This study was supported in part by grants from the Agnes and Knut Mørks foundation, from The Danish Research Agency, The Foundation of 17-121981, The Novo Nordisk Foundation, The Danish Medical Research Council, The Danish Diabetes Association and from The Danish Hearth Foundation.

\section{CONFLICT OF INTEREST}

The authors declare no conflict of interest.

1 Wang C, Crapo LM: The epidemiology of thyroid disease and implications for screening. Endocrinol Metab Clin N Am 1997; 26: 189-218.

2 Pedersen IB, Knudsen N, Jørgensen T, Perrild H, Ovesen L, Laurberg P: Thyroid peroxidase and thyroglobulin autoantibodies in a large survey of populations with mild and moderate iodine deficiency. Clin Endocrinol 2003; 58: 36-42.

3 Brix TH, Hansen PS, Kyvik KO, Hegedüs L: Cigarette smoking and risk of clinically overt thyroid disease: a population based twin case-control study. Arch Intern Med 2000; 160: 661-666. 
4 Brix TH, Hansen PS, Hegedüs L, Wenzel BE: Too early to dismiss Yersinia enterocolitica infection in the aetiology of Graves' disease: evidence from a twin case-control study. Clin Endocrinol 2008; 69: 491-496.

5 Prummel MF, Strieder TGA, Wiersinga WM: The environment and autoimmune thyroid diseases. Eur J Endocrinol 2004; 150: 605-618.

6 Brix TH, Kyvik KO, Hegedüs L: A population-based study of chronic autoimmune hypothyroidism in Danish twins. J Clin Endocrinol Metab 2000; 85: 536-539.

7 Brix TH, Kyvik KO, Christensen K, Hegedüs L: Evidence for a major role of heredity in Graves' disease - a population based study of two Danish twin cohorts. J Clin Endocrinol Metab 2001; 86: 930-934.

8 Phillips DI, Osmond C, Baird J, Huckle A, Rees-Smith B: Is birthweight associated with thyroid autoimmunity? A study in twins. Thyroid 2002; 12: 377-380.

9 Hansen PS, Brix TH, lachine IA, Kyvik KO, Hegedüs L: The relative importance of genetic and environmental effects for the early stages of thyroid autoimmunity. A study of healthy Danish twins. Eur J Endocrinol 2006; 154: 29-38.

10 Brix TH, Petersen HC, lachine IA, Hegedüs L: Preliminary evidence of genetic anticipation in Graves' disease. Thyroid 2003; 13: 447-451.

11 Brix TH, Knudsen GP, Kristiansen M, Kyvik KO, rstavik KH, Hegedüs L: High frequency of skewed $X$-chromosome inactivation in females with autoimmune thyroid disease: a possible explanation for the female predisposition to thyroid autoimmunity. J Clin Endocrinol Metab 2005; 90: 5949-5953.

12 Ozcelik T, Uz E, Akyerli CB et al: Evidence from autoimmune thyroiditis of skewed $\mathrm{X}$-chromosome inactivation in female predisposition to autoimmunity. Eur J Hum Genet 2006; 14: 791-797.

13 Yin X, Latif R, Tomer Y, Davies TF: Thyroid Epigenetics: X chromosome inactivation in patients with autoimune thyroid disease. Ann N Y Acad Sci 2007; 1110: 193-200.

14 Phillips DIW: Twin studies in medical research: can they tell us whether diseases are genetically determined? Lancet 1993; 341: 1008-1009.

15 Morley R, Dwyer T: Studies of twins: what can they tell os about the fetal origins of adult disease? Paediatr Perinat Epidemiol 2005; 19: 2-7.
16 Skytthe A, Kyvik KO, Bathum L, Holm NV, Vaupel JW, Christensen K: The Danish Twin Registry in the new millennium. Twin Res Hum Genet 2006; 9: 763-771.

17 Benyamin B, Sørensen TIA, Schousboe K, Fenger M, Visscher PM, Kyvik KO: Are there common genetic and environmental factors behind the endophenotypes associated with the metabolic syndrome? Diabetologia 2007; 50: 1880-1888.

18 Allen RC, Zoghbi HY, Moseley AB, Rosenblatt HM, Belmont JW: Methylation of Hpall and Hhal sites near the polymorphic CAG repeat in the human androgen-receptor gene correlates with X chromosome inactivation. Am J Hum Genet 1992; 51: 1229-1239.

19 Brunner E: A nonparametric estimator of the shift effect for repeated observations. Biometrics 1991; 47: 1149-1153.

20 Kristiansen M, Knudsen GPS, Bathum L et al: Twin study of genetic and aging effects on X chromosome inactivation. Eur J Hum Genet 2005; 13: 599-606.

21 Naumova AK, Olien L, Bird LM et al: Genetic mapping of X-linked loci involved in skewing of $X$ chromosome inactivation in the human. Eur J Hum Genet 1998; 6: 552-562.

22 Lleo A, Battezzati PM, Selmi C, Gershwin ME, Podda M: Is autoimmunity a matter of sex? Autoimmun Rev 2008; 7: 626-630.

23 Horwitz D, Refetoff S: Graves' disease associated with familial deficiency of thyroxinebinding globulin. J Clin Endocrinol Metab 1977; 44: 242-247.

24 Barbesino G, Tomer Y, Concepcion ES, Davies TF, Greenberg DA: Linkage analysis of candidate genes in autoimmune thyroid disease. II. Selected gender-related genes and the X-chromosome. J Clin Endocrinol Metab 1998; 83: 3290-3295.

25 Imrie H, Vaidya B, Perros P et al: Evidence for a Graves' disease susceptibility locus at chromosome Xp11 in a United Kingdom population. J Clin Endocrinol Metab 2001; 86: 626-630.

26 Brix TH, Kyvik KO, Hegedüs L: Major role of genes in the etiology of simple goiter in females: a population-based twin study. J Clin Endocrinol Metab 1999; 84: 3071-3075. 